\title{
PERFIL DE ADOLESCENTES COM REPETIÇÃO DA GRAVIDEZ ATENDIDAS NUM AMBULATÓRIO DE PRÉ-NATAL ${ }^{1}$
}

\author{
Lia Persona ${ }^{2}$ \\ Antonieta Keiko Kakuda Shimo ${ }^{3}$ \\ Maria Celina Tarallo ${ }^{3}$
}

Persona L, Shimo AKK, Tarallo MC. Perfil de adolescentes com repetição da gravidez atendidas num ambulatório de pré-natal. Rev Latino-am Enfermagem 2004 setembro-outubro; 12(5):745-50.

Este trabalho identificou o perfil biopsicossocial das adolescentes com repetição da gravidez, atendidas num ambulatório de pré-natal. Os dados obtidos através do prontuário médico e entrevista foram analisados quantitativamente. Através dos resultados encontrados e de acordo com a literatura, foram selecionados, do perfil das adolescentes, fatores mais fortemente associados à ocorrência da repetição da gravidez. São eles: menarca precoce, primeira relação sexual após curto intervalo da menarca, repetência escolar, abandono escolar, ausência de ocupação remunerada, baixa renda familiar, envolvimento com parceiros mais velhos, residir com o parceiro, união consensual com o parceiro, um parceiro fixo, baixo uso de condon, história familiar de gravidez na adolescência, ausência do pai por morte ou abandono, reação positiva da família à gravidez anterior, aborto anterior, parto anterior bem conceituado pela adolescente, e ausência à revisão pós-parto anterior.

DESCRITORES: gravidez na adolescência; cuidado pré-natal; enfermagem obstétrica

\section{PROFILE OF ADOLESCENTS WITH REPEATED PREGNANCIES ATTENDED AT A PRENATAL CLINIC}

This study identified the biopsychosocial profile of adolescent with repeated pregnancies, who were attended at a prenatal clinic. Data were collected through patient records and interviews and were subject to quantitative analysis. Based on the obtained results and in accordance with literature, factors that are strongly associated with the occurrence of pregnancy repetition were selected in the adolescents' profiles. These are: early menarche; first sexual intercourse shortly after menarche; school repetition; school dropout; non remunerated occupation; low family income; involvement with older partners; living with the partner; consensual union with the partner; one partner; low condom use; family history of adolescent pregnancy; father's absence because of death or abandonment; positive family reaction to previous pregnancy; previous abortion; adolescent's positive concepts about previous delivery; and absence from previous postpartum consultations.

DESCRIPTORS: adolescent pregnancy; prenatal care; obstetrical nursing

\section{PERFIL DE LAS ADOLESCENTES CON REPETICIÓN DEL EMBARAZO ATENDIDAS EN UN AMBULATORIO DE PRENATAL}

Este trabajo identificó el perfil biopsicosocial de las adolescentes con repetición del embarazo, atendidas en un ambulatorio de prenatal. Los datos obtenidos por medio del prontuario médico y entrevista, fueron analizados cuantitativamente. Por medio de los resultados y de acuerdo con la literatura, fueron seleccionados, entre los perfiles de las adolescentes, factores más fuertemente asociados a la ocurrencia de la repetición del embarazo. Ellos son: menarca precoz; primera relación sexual tras corto intervalo de la menarca; repetición escolar; abandono escolar; ausencia de ocupación remunerada; baja renta familiar; envolvimiento con parejas más viejas; residir con la pareja; unión consensual con la pareja; pareja fija; bajo uso de condón; historia familiar de embarazo en la adolescencia; ausencia del padre por muerte o abandono; reacción positiva de la familia al embarazo anterior; aborto anterior; parto anterior bien conceptuado por la adolescente; y ausencia a la revisión posparto anterior.

DESCRIPTORES: embarazo en adolescencia; atención prenatal; enfermería obstétrica

\footnotetext{
${ }_{1}^{1}$ Pesquisa de Iniciação Científica financiada pela FAPESP; ${ }^{2}$ Graduanda do Curso de Graduação em Enfermagem, e-mail: lia@widesoft.com.br; ${ }^{3}$ Docentes, e-mail: akkshimo@terra.com.br, e-mail: tarallo@fcm.unicamp.br. Faculdade de Ciências Médicas da Universidade Estadual de Campinas
} 
$\boldsymbol{A}$ tecnologia no campo da contracepção e a assistência no âmbito da saúde reprodutiva e sexual têm avançado ultimamente ${ }^{(1)}$, todavia, muitas adolescentes ainda engravidam sem terem planejado sua gestação. "A sociedade como um todo, mostra-se pseudopermissiva, permitindo e estimulando o exercício da atividade sexual dos jovens, proibindo, porém, a gravidez precoce, como se a capacidade reprodutiva pudesse ser analisada de modo isolado e independente da sexualidade"(2).

Embora o desejo consciente ou inconsciente de engravidar seja um dos fatores que levam à gravidez na adolescência $^{(3)}$, ele pode estar sendo influenciado por fatores internos e externos. Um deles é o tipo de relacionamento familiar, especialmente entre pais e filhos, que propicia determinados comportamentos ${ }^{(4)}$. Algumas pesquisas apontam que a maioria das adolescentes que engravidam são filhas de mães que também engravidaram durante a adolescência. Um fenômeno psicológico (inconsciente) de repetição da história materna, podendo ser a gravidez uma tentativa de reconciliação entre mãe e filha $^{(3,5-7)}$

Conforme a literatura, mais da metade das adolescentes engravida por outras causas que não o desejo pela maternidade em si. "Engravidar para não perder o namorado, para sair da casa dos pais e evitar o clima familiar desagradável, para afirmar sua feminilidade através da fertilidade, para encontrar nos cuidados com o filho um objetivo para sua vida, para aplacar a solidão na companhia do filho, etc., por uma vida tortuosa, a tentativa de preencher um vazio interior"(6).

Além desses fatores, a gravidez pode resultar do desconhecimento ou uso inadequado dos métodos contraceptivos, ignorância da fisiologia da reprodução e das conseqüências das relações sexuais, utilização de métodos de baixa eficiência, diminuição da capacidade de julgamento devido ao efeito de bebidas alcoólicas e drogas, entre outros.

Surpreendentemente, um grupo de adolescentes apresenta uma segunda, terceira ou até quarta gestação durante a adolescência, a maioria sem ter planejado ${ }^{(8)}$. Diversos autores constataram altas porcentagens de repetição da gravidez na adolescência (10 a 45\%) acontecendo após pequenos intervalos entre as gestações $(12-24 \text { meses })^{(8-11)}$. "Parece que a primeira gravidez indesejada não é um recurso significativamente forte para prevenir a ocorrência de outras gestações"(2).
A continuação da gravidez entre adolescentes pode levar à desorganização familiar, abandono escolar, afastamento social e do mercado de trabalho, além das questões emocionais. "Mas é importante lembrar que a gestação não programada não implica necessariamente em gravidez indesejada ou em crianças não desejadas. Muitas são rapidamente aceitas ou se transformam ao longo do processo gestacional em, claramente, desejadas, resultando em situações felizes e equilibradas"(2).

Este estudo procurou identificar o perfil biopsicossocial das adolescentes com repetição da gravidez atendidas no ambulatório de pré-natal de adolescentes do CAISM (Centro de Atendimento Integral à Saúde da Mulher) - UNICAMP (Universidade Estadual de Campinas).

\section{MÉTODOS}

Trata-se de um estudo descritivo com abordagem quantitativa realizado no Ambulatório de Pré-Natal de Adolescentes do CAISM. O serviço funciona às terçasfeiras e proporciona assistência pré-natal multiprofissional às gestantes adolescentes. A população foi constituída pelas adolescentes (dos 11 aos 17 anos e 11 meses) agendadas, que estavam no momento da coleta de dados, na segunda ou mais gestação, que compareceram à consulta agendada e que concordaram juntamente com o responsável em participar da pesquisa, através do Consentimento Livre e Esclarecido.

Foram entrevistadas 26 adolescentes durante 0 período de 5 meses de coleta de dados. Entretanto, 8 adolescentes não assinaram o Consentimento Livre e Esclarecido até a data combinada, sendo, portanto, excluídas da pesquisa, incluindo-se, assim, somente 18 adolescentes. Posteriormente, foi feita uma análise informal incluindo os dados das 8 excluídas para verificar se com a inclusão dos dados das mesmas, os resultados sofreriam mudanças. Tal fato não ocorreu, demonstrando que a amostra de 18 adolescentes foi tão válida quanto seria a de 26 adolescentes, se elas tivessem sido incluídas.

A coleta de dados aconteceu após a adolescente ter sido convidada, informada e esclarecida sobre o estudo. Incluiu consulta ao prontuário e entrevista semiestruturada, direcionada por um formulário, num consultório do ambulatório. As perguntas contidas no instrumento de 
coleta de dados, desenvolvido especialmente para essa pesquisa, abrangeram:

- variáveis sociais: idade, grau de escolaridade, abandono escolar, repetência na escola, renda familiar, aspectos relativos à moradia, estado civil nas gestações anteriores e no início da atual, religião, ocupação principal, números de parceiros até o momento, meio de acesso à educação sexual;

- variáveis psicológicas: nota (1-10) para relacionamento com pai, mãe, irmãos e companheiro, nota (1-10) para o desenrolar dos partos anteriores na visão da adolescente, razão do êxodo da escola (se presente), aceitação da família à(s) gravidez(es) anterior(es), planejamento da(s) gravidez(es) anterior(es) e da atual, idade da mãe da adolescente quando (a mãe) engravidou pela primeira vez, cuidador do(s) filho(s) anterior(es) (se vivo), provável cuidador do recém-nascido;

- variáveis biológicas: idade em que ocorreu a menarca, idade ginecológica, idade ao engravidar pela primeira vez, história de aborto(s) anterior(es), número de gestações anteriores levadas a termo ou interrompidas, tipo(s) de parto(s) anterior(es), número de filhos vivos, número de meses entre o último parto ou aborto e o início da gestação atual, presença à última revisão pós-parto, período de amamentação do último filho, utilização, indicação e tipo de método(s) contraceptivo(s) anterior a gestação atual.

O estudo foi aprovado pela Comissão de Ética do CAISM e pelo Comitê de Ética em Pesquisa da Faculdade de Ciências Médicas da Unicamp.

Os dados coletados, e os mesmos inseridos no programa Epi Info 2000 1.0, foram codificados enfocando a freqüência de cada variável. Através da análise quantitativa das porcentagens foi identificado o perfil biopsicossocial das adolescentes.

\section{RESULTADOS}

A idade das adolescentes concentrou-se nos 17 anos (44\%). A idade média da menarca foi 12 anos, o que nos remete a uma idade ginecológica atual de 5 anos para a segunda ou terceira gestação. Todas tiveram sua primeira relação sexual após a menarca, o que ocorreu em média aos 14 anos. A primeira gestação teve maior incidência aos 15 anos (72,22\%).

Quanto à escolaridade, $50 \%$ concluiu o primeiro grau. Felizmente, não foi encontrada nenhuma analfabeta.
Verificou-se que $16,67 \%$ das adolescentes não interromperam os estudos após ficarem grávidas. As que o fizeram, a maioria foi devido à gravidez. A repetência escolar apareceu em $44,44 \%$ das entrevistadas. A escola $(50 \%)$ foi apontada pelas adolescentes como o maior veículo de educação sexual, seguido pela família (16,67\%) e amigos (16,67\%).

O número de parceiros total foi um para $55,56 \%$ das adolescentes, e quatro para 5,56\%. A maior parte das adolescentes era solteira na primeira gestação (66,67\%). Entretanto, no momento da última gestação, apenas $27,78 \%$ se encontravam solteiras e as restantes estavam amasiadas ou casadas, ou seja, em um relacionamento considerado estável socialmente. A idade do parceiro variou dos 17 aos 31 anos, com média nos 23 anos.

A ocupação de $77,78 \%$ das adolescentes é do lar, e a renda familiar da maioria não ultrapassa 5 saláriosmínimos (salário-mínimo no momento da coleta de dados $=R \$ 180)$. Quanto à religião, 44,44\% são católicas, 33,33\% evangélicas e 22,22\% referiram não possuir religião.

Todas as adolescentes moram na área urbana, em casas pertencentes, na maioria, à família. Predominantemente, residem com o parceiro $(77,78 \%)$. O número de pessoas que moram na mesma casa é de 2 a 4 para $66,67 \%$ das adolescentes, em 1 a 4 cômodos para $61,11 \%$. Todas as casas possuem água encanada e energia elétrica. Não há asfalto na rua de $33,33 \%$ das adolescentes.

A pílula foi o método contraceptivo mais utilizado pelas adolescentes $(55,55 \%)$, durante o período entre o último parto ou aborto e o início da gestação atual, seguido pelo hormônio injetável (22,23\%). Somente $27,78 \%$ das adolescentes utilizaram o condon. A ausência de método contraceptivo apareceu em $11,11 \%$ das adolescentes. $O$ grau de escolaridade não influenciou o uso do contraceptivo que foi indicado para a maioria, pelo profissional médico (82,35\%).

A maioria das mães das adolescentes (64,71\%) teve a sua primeira gravidez dos 16 aos 19 anos. Entretanto, o teste Exato de Fisher demonstrou valores não significativos para afirmar uma associação nesta pesquisa. A própria adolescente apareceu como principal cuidadora dos filhos vivos e provável cuidadora do filho em gestação. A taxa de ausência de amamentação do filho anterior foi de $5,56 \%$.

O conceito, de um a dez, apontado pelas 
adolescentes para o relacionamento com a mãe foi na maioria, dez. Já com o pai, foi conceito zero, significando, nessa pesquisa, a ausência do familiar por morte ou desaparecimento. Dentre as adolescentes que têm pai ausente, $87,5 \%$ estão amasiadas ou casadas. Para com os irmãos, de uma forma geral, o conceito não foi menor do que cinco. Para com o parceiro, foi na maioria, dez. A reação da família à primeira gestação e à atual foi predominantemente de amparo.

Dentre as adolescentes, $66,67 \%$ referiram não ter planejado a primeira gestação e $77,78 \%$ que não planejaram a gestação atual. Nenhuma adolescente em estado civil não estável planejou a gestação atual. Somente metade alcançou uma gestação a termo. A maioria, $88,89 \%$, está na segunda gestação, $5,56 \%$ na terceira e $55,56 \%$ não tem filhos vivos. Os partos anteriores foram predominantemente vaginais e bem conceituados pelas adolescentes.

O intervalo entre o último parto ou aborto e a concepção da gestação atual foi para $50 \%$, de 6 a 12 meses. Do total, $11,11 \%$ apresentou nova gestação num intervalo menor que 6 meses. Mais da metade $(55,52 \%)$ apresentou pelo menos um aborto prévio. Apenas 22,22\% compareceu à última revisão pós-parto.

\section{DISCUSSÃO}

Os dados numéricos como a média da menarca na faixa dos 12 anos, primeira relação sexual dos 12 aos 15 anos, primeira gestação aos 15 anos e história de apenas um parceiro para a maioria, igualaram-se aos da literatura ${ }^{(7-8,12-14)}$. O dado a respeito do número de parceiros é visto como positivo, pois demonstra a ausência de promiscuidade entre as adolescentes.

Quanto à situação marital, os achados revelam grande número de uniões consensuais durante a gravidez atual, embora muitas dessas adolescentes possam ter "regularizada" a sua situação marital durante a gestação atual e não anterior a essa. Entretanto, um dos fatores de risco para a repetição da gravidez é estar morando com um parceiro ou casada, situação em que a maioria das adolescentes estavam no momento da entrevista ${ }^{(15)}$. Isso também ocorreu em outros estudos ${ }^{(4,8,10,13,16-17)}$. Outro dado que a literatura aponta como fator associado é a idade do parceiro, geralmente maior, achado também obtido nesta pesquisa $^{(7,10)}$.
Obteve-se valores positivos de grau de escolaridade em relação à literatura ${ }^{(4,7-8,13-14,16)}$. Quanto ao grande número de abandono escolar, resultante da gravidez entre as adolescentes, o mesmo dado foi constatado em vários estudos relacionados ao tema ${ }^{(10,17)}$. Constatou-se também presença de repetência escolar que pode ser um fator de risco à repetição da gravidez na adolescência, pela experiência escolar negativa que traz ${ }^{(11)}$. A primeira gravidez levou ao abandono escolar, o que favoreceu outras gravidezes, principalmente se não houve retorno à escola em seis meses após a primeira gravidez $^{(15)}$.

Estudos anteriores encontraram valores de renda familiar inferiores a 5 salários-mínimos (valor da época dos estudos), concordes a esta pesquisa, 5 salários-mínimos (valor no momento da coleta de dados deste estudo) ${ }^{(7,13)}$. Entretanto, as adolescentes não contribuem com essa renda, sendo dependentes da família ou do parceiro. As que possuem filhos vivos ocupam-se cuidando dos mesmos, já que a própria adolescente apareceu como principal cuidadora dos filhos e provável cuidadora do filho em gestação, como já descrito ${ }^{(13)}$.

A situação socioeconômica das adolescentes, estimada pela renda familiar, e o número de dependentes, além da presença de água encanada, luz elétrica e asfalto na rua, indicam que essas provêm de classe média baixa e algumas de classe baixa.

Quanto à religião, o catolicismo predominou assim como em outras pesquisas ${ }^{(13-14)}$. A reação positiva da família em relação à(s) gravidez(es) anterior(es) foi um dado encontrado nesse estudo e que pode ser fator contribuinte à repetição da gravidez ${ }^{(11,18)}$.

Um dado interessante que apareceu neste estudo, não verificado por outros na literatura, foi a ausência do pai da adolescente por morte ou desaparecimento. Esse dado denota a ausência da figura masculina paterna na vida da adolescente. A maioria das adolescentes com pai ausente encontrava-se amasiada ou casada, o que poderia significar a busca pela figura masculina ausente.

Assim como em outras pesquisas, nem todas utilizaram algum tipo de contraceptivo ${ }^{(3,5,7,13-14)}$. Entretanto, a porcentagem não foi tão baixa como em alguns casos da literatura ${ }^{(12)}$, provavelmente devido ao fato de todas serem multigestas e já terem passado por um serviço de saúde voltado para a saúde reprodutiva com orientações específicas.

A pílula, em conformidade com a literatura, 
também foi relatada como o contraceptivo mais utilizado entre as adolescentes ${ }^{(8,12)}$. Um estudo demonstrou menor repetição da gravidez naquelas que fizeram uso do contraceptivo injetável ${ }^{(19)}$. Já o uso do condon deixou a desejar, visto ser a única maneira de prevenir as doenças sexualmente transmissíveis.

Os dados relativos ao fato da mãe da adolescente também ter tido uma gravidez na adolescência não foram significativos, mas a literatura aponta para a repetição dos modelos familiares como fator associado ${ }^{(3,5-7)}$.

Assim como em outros estudos, muitas adolescentes não planejaram suas gestações ${ }^{(5-8,14)}$ e a maioria estava na segunda gestação ${ }^{(8,20)}$. O intervalo entre o último parto ou aborto e a concepção da gestação atual também foi inferior a 12 meses $^{(8,10,17,19-20)}$.

Quanto às ocorrências de aborto, um estudo constatou $40,74 \%$ de abortos prévios ${ }^{(7)}$ e outro estudo, $30 \%{ }^{(17)}$, valores abaixo dos encontrados neste estudo, no qual mais da metade das adolescentes $(55,52 \%)$ referiu a ocorrência de pelo menos um aborto espontâneo prévio. Entretanto, não se investigou outras fontes, além do relato das adolescentes, para comprovar se não houve realmente nenhum aborto provocado. Assim, não existe a possibilidade de afirmar seguramente o que levou aos abortos prévios, ficando tal questionamento para estudos posteriores. Dentre as que não abortaram, houve maior incidência de parto vaginal. A literatura traz números equilibrados de parto vaginal ${ }^{(8)}$, e de parto cesariana ${ }^{(14)}$ entre adolescentes. A maioria dos partos vaginais foram conceituados pelas adolescentes com notas altas, o que significa que na percepção delas a experiência do parto foi positiva.

\section{CONCLUSÕES}

A presença marcante de alguns fatores no perfil das adolescentes com repetição da gravidez, pesquisadas

\section{REFERÊNCIAS BIBLIOGRÁFICAS}

1. Mandu ENT. Trajetória assistencial no âmbito da saúde reprodutiva e sexual: Brasil, século XX. Rev Latino-Am Enfermagem 2002 maio-junho;10(3):358-71.

2. Pinto e Silva JL. Gravidez na adolescência: desejada $X$ não desejada. Femina 1998; 26(10):825-30.

3. Correa MM, Coates V. Implicações sociais e o papel do pai. In: Maakaroun MF, Souza RP, Cruz AR. Tratado de Adolescência. Rio de Janeiro (RJ): Cultura Médica; 1991. p.408-15. no presente estudo, demonstra que existe influência causal multifatorial por trás do evento. Esses dados corroboram com os encontrados na literatura: menarca precoce (até os 12 anos), primeira relação sexual após curto intervalo da menarca, repetência escolar, abandono escolar, ausência de ocupação remunerada, baixa renda familiar, envolvimento com parceiros mais velhos, residir com o parceiro, união consensual com o parceiro, um parceiro fixo, baixo uso de condon, história familiar de gravidez na adolescência (pela mãe, irmãs, primas), ausência do pai por morte ou abandono, reação positiva por parte da família à gravidez anterior, aborto anterior, parto anterior bem conceituado pela adolescente e ausência à revisão pós parto-anterior. Embora significativos, os resultados são limitados a essa população, sendo sugestivo um futuro aprofundamento quantitativo e qualitativo, com estudo de cada dado isoladamente.

O perfil identificado afirma a importância de programas, alicerçados na literatura, dirigidos aos adolescentes, como dispor novas formas, que não a maternidade, de saciar as necessidades emocionais e de desenvolvimento através de atividades técnicas e/ou práticas pela educação alternativa, programas de treinamento vocacional e elaboração de projeto de vida. Também são importantes os programas educativos sobre desenvolvimento sexual, treinamento de habilidades interpessoais, de negociação e de comunicação nas escolas, além de desenvolver instrumentos para identificar adolescentes com alto risco para a gravidez precoce. Ainda existe a necessidade de disponibilizar espaços, incluindo a internet, para os jovens falarem e colocarem na palavra o que sentem, e não nos seus atos. Finalmente, o envolvimento dos pais, professores e profissionais da saúde (especialmente aqueles ligados ao Programa de Saúde da Família, Programa de Atendimento Integral à Saúde da Mulher e serviços de pré- e pós-natal) é essencial para o sucesso de qualquer ação direcionada aos adolescentes.

4. Guijarro S, Naranjo J, Padilla M, Gutiérez R, Lammers C, Blum RW. Family risk factors associated with adolescent pregnancy: study of a group of adolescent girls and their families in Ecuador. J Adolesc Health 1999; 25(2):166-72.

5. Schiller R. Gravidez na adolescência: uma questão de hereditariedade. Pediatr Mod 1994; 30(6):984-6.

6. Moreira CC. Fique alerta quando: a adolescente engravida. Pediatr Mod 1997; 33(5):320.

7. Abdallah VOS, Mayrink L, Gonsalves RMP, Nishioka SA. Gravidez na adolescência: experiência em um hospital universitário. Pediatr Mod 1998; 34(9):561-70. 
8. Pinto e Silva JL, Nogueira CWM. A multigravidez na adolescência. In: Organização Panamericana da Saúde e OMS. Coletânea sobre saúde reprodutiva do adolescente brasileiro. Brasília (DF): Organização Panamericana da Saúde/OMS; 1988. p.101-11.

9. Rubin V, East PL. Adolescents' pregnancy intentions. J Adolesc Health 1999; 24(5):313-30.

10. Pfitzner M, Hoff C, Mcelligott K. Repeat pregnancy in program for pregnant and parenting teens: 15 years of experience. J Adolesc Health 2001 February; 28(2):129.

11. Coard S, Nits K, Park P, Felice M. Predictors of repeat pregnancy in urban adolescent mothers. [abstract]. J Adolesc Health 1998 February; 22(2):173.

12. Schor N, Lopes AF. Adolescência e anticoncepção: estudo de conhecimento e uso em puérperas internadas por parto ou aborto. Rev Saúde Pública 1990; 24(6):506-11.

13. Maia NL Filho, Mathias L, Tedesco RP, Cesared MD, Herculano MA, Porta RMP. Gravidez entre adolescentes precoces: um evitável problema social. J Bras Ginecol 1994 outubro; 104(10):363-7.

14. Frazão ZS, Martins MG. Perfil da adolescente no ciclo grávido-puerperal. Femina 1997; 25(5):419-30.

15. American Academy of Pediatrics Committee on Adolescence and Committee on Early Childhood, Adopting, and Dependent Care. Care of adolescent parents and their children. Pediatrics 2001 February; 107(2):429-34.

16. Coates V, Correa MM. O atendimento de adolescentes grávidas. In: Maakaroun MF, Souza RP, Cruz AR. Tratado de Adolescência. Rio de Janeiro (RJ): Cultura Médica; 1991. p.390-407.

17. Stevens-Simon C, Kelly L, Singer D. Absence of negative attitudes toward childbearing among pregnant teenagers - a risk factor for a rapid repeat pregnancy? Arch Pediatr Adolesc Med 1996; 150:1037-43.

18. Godinho RA, Schelp JRB, Parada CMGL, Bertoncello NMF. Adolescentes e grávidas: onde buscam apoio? Rev LatinoAm Enfermagem 2000 abril; 8(2):25-32.

19. Desai A, Wiemann C, Berenson A. Ethnic differences in rates of contraceptive continuation and repeat pregnancy among adolescent mothers at 12 and 24 months postpartum. [research presentations]. J Adolesc Health 2001 February; 28(2):100.

20. Mariotoni GGB, Barros AA Filho. A gravidez na adolescência é fator de risco para o baixo peso ao nascer? J Pediatria 1998; 74(2):107-13. 